\title{
Emprego das Ferramentas de Abordagem Familiar na Estratégia Saúde da Família em Montes Claros, MG: relato de caso
}

\author{
Use of Family Approach Tools in the Family at Primary Healthcare in Montes Claros, MG: \\ case report
}
Uso de Herramientas de Abordaje Familiar en el Nivel de Atención Primaria de Salud de La Familia en Montes Claros, MG: relato de caso

Cláudio Wagnus Xavier Lopes Júnior ${ }^{1 *}$, Rayssa de Luar Oliveira Dias Teixeira ${ }^{1}$, Verônica Cardoso de Abreu${ }^{1}$, Luis Paulo Morais Farias ${ }^{1}$, Samara Ferreira Gomes ${ }^{1}$, Carla Patrícia Martins Cardoso², Jéssica Rejane Durães Soares ${ }^{1}$, Vanessa Cristiane Araújo Oliveira ${ }^{1}$, Mayara Karoline Silva Lacerda ${ }^{1}$, Marianne Silva Soares ${ }^{2}$.

\section{RESUMO}

Objetivo: Relatar a experiência de aplicação de ferramentas de abordagem familiar em uma família cadastrada em uma equipe da Estratégia de Saúde da Família do município de Montes Claros, Minas Gerais, Brasil. Detalhamento do Caso: Trata-se de um estudo descritivo, longitudinal, de caráter qualitativo. Foram aplicadas as ferramentas de abordagem familiar: Genograma, Ecomapa, Ciclo de Vida, FIRO e P.R.A.C.T.I.C.E., bem como uma Conferência familiar para a apresentação e discussão das propostas de intervenções planejadas pela equipe de saúde diante de uma situação de vulnerabilidade social, demanda excessiva pelo serviço de saúde e dificuldade no controle de doenças crônicas. Considerações Finais: $O$ emprego das ferramentas corroborou na compreensão da estrutura, funcionamento e dinâmica da família, aproximando os profissionais de saúde da família em estudo. Essa experiência permitiu que a equipe contribuísse na reorganização dos papéis familiares, encorajando a redistribuição de tarefas entre os integrantes, através de um plano de cuidados elaborado a partir das demandas da família.

Palavras-chave: Estratégia de Saúde da Família, Atenção Primária à Saúde, Relações Familiares, Atenção à saúde, Comunicação Interdisciplinar.

\begin{abstract}
Objective: To report the experience of applying family approach tools on a family enrolled in a Family at primary healthcare team from the city of Montes Claros, Minas Gerais, Brazil. Case Details: This is a descriptive, longitudinal, qualitative study. The family approach tools that were applied: Genogram, Ecomap, Life Cycle, FIRO and P.R.A.C.T.I.C.E., and then a family conference was held to present and discuss the proposals for interventions planned by the health team in the face of a situation of social vulnerability, excessive demand for the health service, difficulty in controlling chronic diseases. Final Considerations: The use of the tools corroborated to the understanding of the structure, functioning and dynamics of the family, bringing health professionals closer to the family under the study. This experience allowed us to contribute to the reorganization of family roles by encouraging the redistribution of tasks among the members through a plan of care elaborated from the demands of the family.
\end{abstract}

Keywords: Family Health Strategy, Primary Health Care, Family relationships, Health care, Interdisciplinary Communication.

1Universidade Estadual de Montes Claros (UNIMONTES), Montes Claros-MG.

22Secretaria Municipal de Saúde - Montes Claros/MG. *E-mail: claudiowagnus13@gmail.com

SUBMETIDO EM: 4/2019

ACEITO EM: 5/2019

PUBLICADO EM: 8/2019 


\section{RESUMEN}

Objetivo: Reportar la experiencia de aplicación de las herramientas del enfoque familiar en una familia registrada en el nivel de atención primaria de la Municipalidad de Montes Claros, Minas Gerais, Brasil. Detalles: este es un estudio descriptivo, longitudinal, cualitativo carácter. Fueron aplicadas las herramientas de enfoque familiar: Genogram, Ecomapa, ciclo de vida, herida y P.R.A.C.T.I.C.E., así como una familia planificada Conferencia para la presentación y discusión de las intervenciones propuestas por el equipo de salud antes de un situación de vulnerabilidad social, excesiva demanda de servicios de salud y dificultad en el control de las enfermedades crónicas. Reflexiones finales: el trabajo acompañados de herramientas en la comprensión de la estructura, función y dinámica de la familia, los profesionales de la salud de la familia. Esta experiencia permitió al equipo de contribuir en la reorganización de roles familiares, fomentar la redistribución de tareas entre los miembros, a través de un plan de atención de las demandas de la familia.

Palabras clave: Familia Estrategia de salud, Atención Primaria de Salud, las relaciones familiares, Salud, Comunicación interdisciplinaria.

\section{INTRODUÇÃO}

Desde o ano de 1994 o Brasil tem cursado transições pelo cenário de saúde pública, como a implantação da Estratégia de Saúde da Família (ESF) pelo Ministério da Saúde, que objetiva a reorientação do modelo assistencial, em que o enfoque do cuidado e atenção à saúde é voltado para a família. Então, são ofertadas ações de promoção, proteção, prevenção, recuperação e reabilitação aos usuários do Sistema Único de Saúde (SUS). Ademais, quando o acompanhamento se torna insuficiente para atender às demandas dos clientes, estes são direcionados aos serviços especializados. Entretanto, a ESF permanece responsável pela coordenação destes usuários, uma vez que retornam sempre para o cuidado da ESF após os exames e consultas especializadas. Dentro da proposta, cada ESF possui uma equipe multiprofissional, que trabalha com um número definido de famílias, estabelecido através da delimitação do território adstrito, decretado pela Portaria Nacional de Atenção Básica (PNAB) (BRASIL, 2010; BRASIL, 2017; OLIVEIRA PS et al., 2016).

Compreende-se por família um grupo de pessoas unidas através de laços consanguíneos ou não, que convivem no mesmo domicílio e compartilham dos mesmos interesses, em que há trocas afetivas. É onde se constrói a identidade e estabelece o senso de pertencimento (SANTOS KKF et al., 2015; OLIVEIRA VCA et al., 2017).

Nesse ínterim, torna-se imprescindível que a $A B$ utilize ferramentas de abordagem familiar para proporcionar uma visão ampliada do contexto biopsicossocial-espiritual em que a família está inserida, uma vez que cada uma possui suas particularidades e diferentes arranjos. Isso permite a realização de um cuidado mais assertivo por ser planejado junto aos indivíduos que compõem a família, de acordo com suas especificidades e levando em consideração a compreensão como um todo dos problemas levantados (OLIVEIRA VCA et al., 2017).

A abordagem familiar requer uma relação de respeito e confiança, sendo assim, quanto maior o vínculo entre a equipe e família, melhor é a aceitação das ações pelos indivíduos e, por conseguinte, os resultados apresentados. As ferramentas de abordagem familiar são utilizadas através do desdobramento de algum problema relevante, que causa repercussões individuais e/ou familiares, tais como: procura exacerbada pelo serviço de saúde, apresentação de sintomas psicossomáticos, apresentação de alterações comportamentais graves, dificuldade no acompanhamento de doenças crônicas, insuficiência do modelo médico na resolução de demandas familiares e ocorrência de óbito intrafamiliar. Dentre as ferramentas mais utilizadas para a compreensão e intervenções familiares, estão: Genograma, Ecomapa, FIRO, Ciclo de vida, P.R.A.C.T.I.C.E e Conferência familiar (BRASIL CHG, 2010; SILVA JV e SANTOS SMR, 2003).

O Genograma apresenta o contexto familiar em relação a parentescos e doenças prevalentes. $O$ Ecomapa representa o contexto social em que a família está inserida, o apoio familiar e relações. A ferramenta FIRO abrange as especificidades dos relacionamentos intrafamiliares. O ciclo de vida mostra em 
qual fase a família encontra-se, como: adulto jovem independente, casamento, nascimento do primeiro filho, família com filhos pequenos, família com filhos adolescentes, lançando os filhos e seguindo em frente, aposentadoria e famílias no estágio tardio (a velhice). O P.R.A.C.T.I.C.E aborda em oito etapas o desenrolar dos problemas de relacionamentos familiares (LACERDA MKS et al., 2017; MELLO DF et al., 2005; PEREIRA APS et al., 2009).

O presente trabalho objetiva relatar o estudo de caso realizado em uma família residente na área de abrangência da ESF Jasmim, no município de Montes Claros- MG, utilizando ferramentas de abordagem familiar.

\section{DETALHAMENTO DO CASO}

Trata-se de um estudo descritivo, longitudinal, de caráter qualitativo, realizado pela equipe de Residência Multiprofissional em Saúde da família, na área de abrangência da ESF Jasmim, no município de Montes Claros-MG. Ocorreu entre setembro de 2018 e março de 2019, após aprovação do Comitê de Ética em Pesquisa da Universidade Estadual de Montes Claros, pelo parecer no 572.244 de 2014. Para tal, foram realizadas quatro visitas domiciliares, três reuniões com a família na Unidade e três reuniões intersetoriais.

A escolha da família deu-se devido à demanda excessiva pelo serviço de saúde, dificuldade no controle de doenças crônicas e insuficiência do modelo médico. Através da análise do contexto familiar, foram elaborados o Genograma, Ecomapa, Ciclo de vida, F.I.R.O. e P.R.A.C.T.I.C.E. Para consolidação das informações, realizou-se uma Conferência familiar para a apresentação e discussão das propostas de intervenções planejadas pela equipe de saúde. Para manutenção do sigilo, foram utilizadas apenas as iniciais dos nomes e idades dos componentes da respectiva família.

A paciente índice Luzia, 40 anos, nascida em Montes Claros, possui ensino fundamental incompleto (4 $4^{\mathrm{a}}$ série), trabalha como diarista de duas residências em bairros adjacentes, reside com seus sete filhos e a nora em casa cedida pelos irmãos como parte de herança do pai. A residência possui sete cômodos, sendo três quartos, cozinha, sala e dois banheiros, em condições precárias, mas que passa por uma reforma conduzida por ação social da igreja.

Luzia possui quatro filhos, Gustavo (20 anos), Júlio (17 anos), Paula (15 anos), Rafaela (9 meses) do seu primeiro relacionamento com Carlos, o qual encontra-se em união estável atualmente. Carlos reside na zona rural, trabalha como "caseiro" e não possui contato frequente com a família, disponibilizando ajuda financeira de maneira irregular. Em um segundo relacionamento com Mário, Luzia teve três filhos Ester (11 anos), Carolina (9 anos), Lucas (6 anos) e um aborto espontâneo. A ruptura de tal relacionamento se deu devido à turbulência na relação do casal.

Luzia possui quatro irmãos, Marcos, Joana, Helena e Márcio, sendo a irmã Helena e o irmão Márcio as pessoas com maior vínculo e confiança. O pai João faleceu de cardiopatia devido a Doença de Chagas aos 70 anos, e a mãe Ana faleceu de infarto.

O estabelecimento de vínculo entre a ESF e a família auxilia no cumprimento das diretrizes da PNAB 2017. Dentre elas, destaca-se a longitudinalidade, em que a equipe de saúde responsável consegue manter a assistência pelo contato maior com a família, adquirindo mais consentimento para a realização de intervenções. Como forma de aumentar o vínculo com a família e auxiliar financeiramente, a equipe de saúde realiza campanha de arrecadação de alimentos e roupas quando solicitados por Luzia.

A ESF Jasmim realizou acompanhamento pré-natal de Luzia da sua última gestação, sendo necessário encaminhamento ao pré-natal de alto risco devido à hipertensão arterial sistêmica (HAS) descompensada, por controle ineficaz do tratamento medicamentoso pela mesma e infecção por toxoplasmose na gestação. Nesse momento verificou-se a dificuldade de Luzia em aderir ao tratamento e acompanhamento no alto risco. Após o nascimento, a filha Rafaela é acompanhada no Centro de Referência em Doenças Infecciosas (CERDI) e nas consultas de puericultura pela Enfermeira e Pediatra. 
O filho Lucas, que após sofrer um atropelamento apresenta dislalia, necessita de acompanhamento com neurologista e fonoaudiólogo. A equipe identificou que, apesar da necessidade de acompanhamento dos filhos, a mãe, Luzia, não manifesta interesse e disponibilidade relacionados a esse acompanhamento.

Também foi realizado o pré-natal odontológico de Luzia, atendimento odontológico dos filhos e da nora, Renata, além de responsabilizar a família sobre o autocuidado da saúde bucal. Porém, há pouca adesão dos mesmos.

Por ser observado durante o acompanhamento que existe vulnerabilidade social na família, foi solicitado pela ESF Jasmim a avaliação e parceria do Centro de Referência de Assistência Social (CRAS) para intervenções. Então foi realizado o cadastro da mesma pelo CRAS para acesso a frutas, verduras e legumes distribuídas gratuitamente na instituição. Além disso, os adolescentes da casa foram convocados a participarem dos cursos e capacitações oferecidos para auxílio ao $1^{\circ}$ emprego formal.

Figura 1 - Genograma da Família de Luzia, Montes Claros, 2019.

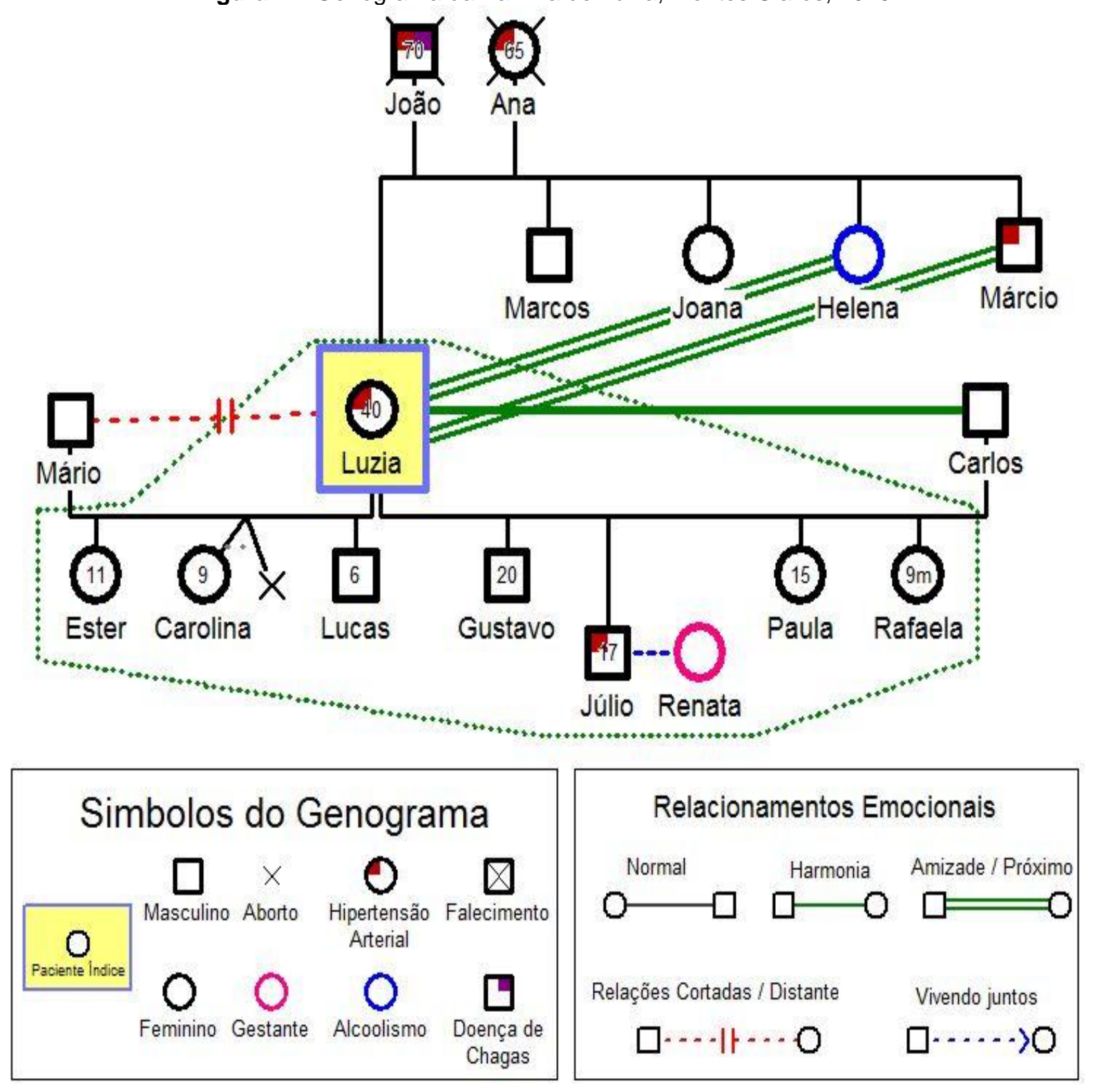

Fonte: Elaborado pelos autores LOPES JÚNIOR CWX, TEIXEIRA RLOD, ABREU VC, FARIA LPM, GOMES SF, CARDOSO CPM, SOARES JRD, OLIVEIRA VCA, LACERDA MKS, SOARES MS. 
Figura 2 - Ecomapa da Família de Luzia, Montes Claros, 2019.

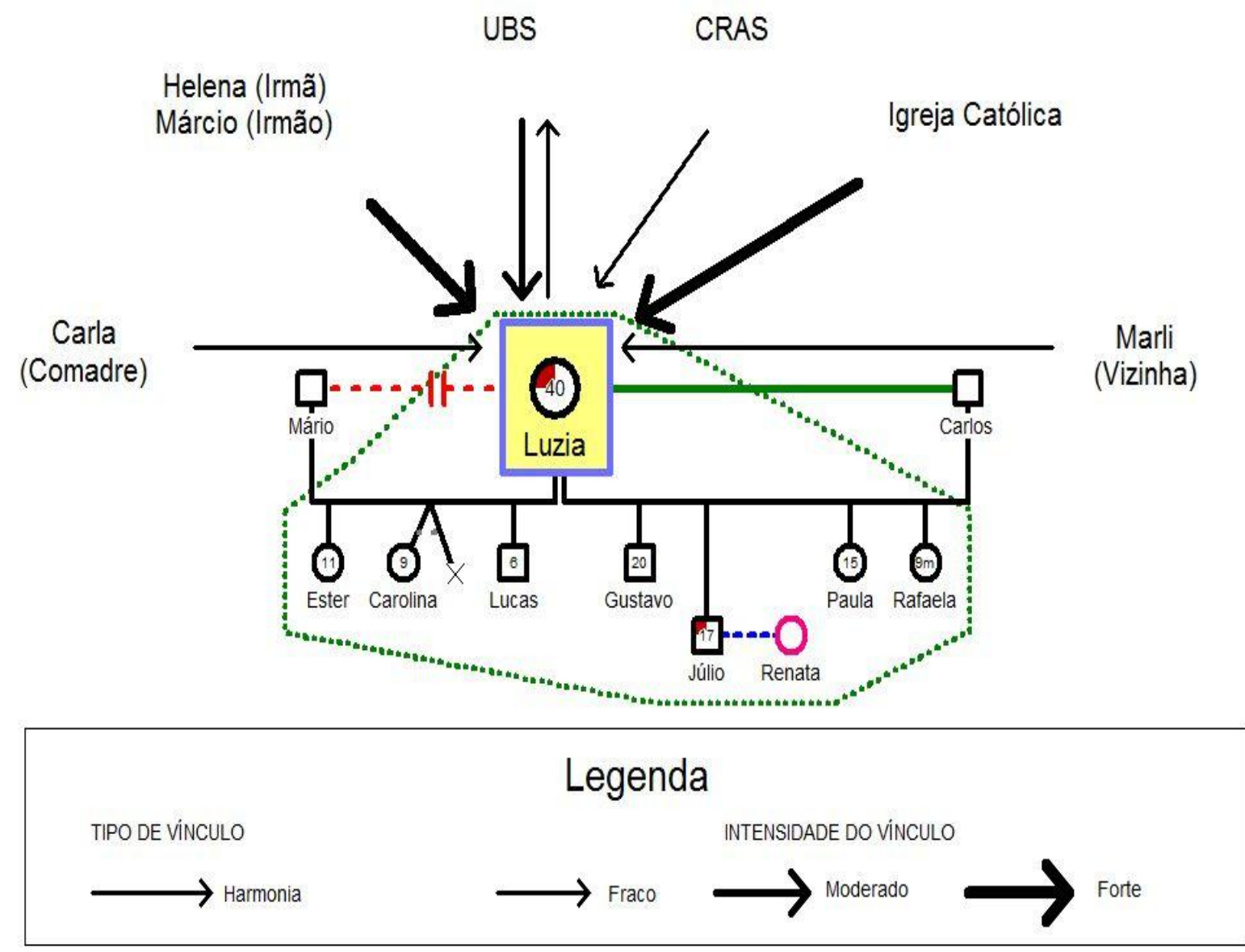

Fonte: Elaborado pelos autores LOPES JÚNIOR CWX, TEIXEIRA RLOD, ABREU VC, FARIA LPM, GOMES SF, CARDOSO CPM, SOARES JRD, OLIVEIRA VCA, LACERDA MKS, SOARES MS.

\section{DISCUSSÃO}

O Genograma consiste numa representação gráfica da família de forma cronológica, que abrange várias gerações, morbidade e causas de mortalidade, relacionamentos e suas especificidades como mostram a (Figura 1) (FELÍCIO LFF et al., 2018; PEREIRA APS et al., 2009).

No Ecomapa são representados os relacionamentos e grupos de apoio familiar e a intensidade da relação entre estes, fornecendo uma visão ampliada da família. É uma ferramenta que ajuda a conectar as circunstâncias ao meio ambiente, tornando-se possível a visualização de uma "fotografia" das principais relações que a família tem com o ambiente (Figura 2) (LACERDA MKS et al., 2017; MELLO DF et al., 2005; PEREIRA APS et al., 2009).

A integração intersetorial é imprescindível quando os problemas levantados ultrapassam o poder de resolução da ESF, tornando-se parceiros em prol da melhoria da qualidade de vida dos indivíduos (DIAS MAS et al., 2014).

O Ecomapa da família em estudo evidencia que o CRAS fornece auxílio à família com distribuição de frutas, verduras, legumes, capacitações para o $1^{\circ}$ emprego formal dos adolescentes. Luzia possui vínculo forte com dois irmãos Helena e Márcio, além de se relacionar com Marli (vizinha) e Carla (Comadre). Há forte ligação com a Igreja Católica do bairro, pois estes prestam assistência social e financeira à família. Percebe-se que Luzia possui uma relação de proximidade com a UBS e vê o serviço como principal recurso para a resolução de problemas. 
O Ciclo de Vida Familiar é uma ferramenta que identifica a fase de vida em que os componentes se encontram, de forma a abordar os problemas prevalentes em cada fase, apontando a melhor forma de resolução (BRASIL, 2011; SILVA JV e SANTOS SMR, 2003). O núcleo familiar se encaixa nos ciclos: Família com filhos pequenos e família no estágio tardio. Neste estágio, a mulher em idade fértil e/ou com filhos pequenos se torna avó. Isto traz repercussões na família, que se depara com o aumento das responsabilidades, despesas e alterações no funcionamento de maneira geral.

O FIRO (Fundamental Interpersonal Relations Orientations) é uma ferramenta pautada pelos sentimentos envolvidos nas relações intrafamiliares. Dentro da abordagem, encontram-se os tópicos: inclusão, referente à interação entre os membros; controle-relações de poder/autoridade; intimidade - como acontece o desenrolar dos relacionamentos (BRASIL CHG, 2010; NOBRE LLR et al., 2014). No contexto de relações de poder, o segundo filho mais velho, Júlio, possui o papel de autoridade, substituindo a figura paterna, auxiliando na educação dos mais novos. Quanto à inclusão/interação e intimidade, a nora da paciente índice, Renata, e sua filha, Paula, possui relacionamento próximo e harmonioso. A mãe e as crianças possuem forte ligação, justificado pelo afeto materno, demonstrando grande dependência.

A ferramenta P.R.A.C.T.I.C.E. (Problem, Roles, Affect, Communication, Time of life cycle, Illness, Copingwith stress, Ecology) refere-se a uma descrição dos problemas da família de forma esquematizada (DITTERICH RG et al., 2009; FERNANDES CLC e CURRA LCD, 2006). Esta ferramenta permite a compreensão da família por meio da identificação de oito itens:

- Problems (Problemas): Dentre os problemas levantados, encontrou-se negligência quanto ao tratamento medicamentoso de Luzia, Júlio e acompanhamento especializado da filha Rafaela e Lucas, ausência nas consultas agendadas e vulnerabilidade social.

- Roles and Structure (Papéis e estrutura familiar): Notou-se que devido à falta de divisão de funções, a paciente índice tem se sobrecarregado, o que dificulta a realização do seu papel específico de mãe. $\mathrm{Na}$ família, o filho Júlio substitui o papel do pai devido a sua ausência frequente, a filha Ester conhece o estado de saúde da família melhor que a mãe e busca tentar resolvê-lo. Os filhos Júlio e Gustavo, apesar de apresentarem requisitos compatíveis com o mercado de trabalho, não buscam empregos que auxiliem no sustento da casa. Há ainda pequenas atividades, como os afazeres da casa e auxílio das tarefas escolares, em que há a necessidade de auxílio pelos vizinhos, pois não há proatividade dos adolescentes.

- Affect (Afetar): Apesar das dificuldades apresentadas, nenhum dos componentes identifica como problemas as questões abordadas pelos profissionais de saúde. Nota-se que a família consegue sobressair em meio às dificuldades apresentadas, demonstrando ter acostumado com os problemas vivenciados.

- Communication (Comunicação): Verificou-se que há dificuldade quanto à comunicação com o esposo de Luzia, uma vez que este não está presente na rotina da família. Ocorre também uma falha quanto à paciente não conseguir estabelecer uma comunicação em que os filhos se submetam às suas ordens. Dessa forma, as tarefas da família são negligenciadas pelos filhos.

-Time of life cycle (Tempo no ciclo de vida familiar): Dentre os vários ciclos de vida que a compõem, destaca-se o de família com filhos pequenos, em que há dependência das crianças em todos os aspectos, exigindo assim, maiores cuidados por parte da mãe. Desse modo, ocorre uma interferência nas demais tarefas domésticas e vida profissional de Luzia.

- Illness: (Doenças na família ocorridas no passado e presente): Luzia é portadora de HAS, bem como o filho Júlio. O filho Lucas possui dislalia e Rafaela necessita de acompanhamento rigoroso com infectologista, sendo negligenciado pela família. Os filhos menores tiveram doenças respiratórias agudas. Os pais do paciente índice faleceram devido a cardiopatias e o irmão Márcio também é cardiopata. Observou-se que apesar de haver tratamento e/ou prevenção de determinadas doenças, as mesmas são negligenciadas pela família.

- Coping with stress (Lidando com o estresse): Observou-se que há dificuldade de os membros assumirem tarefas para minimizar o trabalho da paciente, o que prejudica nas demais tarefas que exigem 
mais comprometimento, como as questões de saúde e financeiros. Sendo assim, a mesma sempre recorre ao apoio comunitário, até por simples afazeres, como ensinar tarefas escolares para as crianças.

- Ecology (Meio ambiente): A família procura apoio nos setores expostos no ECOMAPA (ESF, CRAS, Igreja e amigos/familiares) para auxílio na resolução de problemas financeiros, internações, consultas, dentre outros, para encontrar uma solução.

A Conferência Familiar consiste numa reunião realizada junto à família, com o propósito de auxiliar na resolução dos problemas, explanando as possíveis soluções e conscientizando quanto à importância da reorganização para melhoria da qualidade de vida dos mesmos (BRASIL, 2011; SILVA JV e SANTOS SMR, 2003). Através da reunião com os membros da família, foram levantados os problemas encontrados, como a sobrecarga da paciente índice, devido à falta de divisão de tarefas (cuidados com o lar, com as crianças menores, ensino da tarefa escolar, a busca de verduras no CRAS, sobre as consultas das crianças que necessitam de acompanhamento especializado), a necessidade da busca de emprego formal pelos filhos mais velhos e sobre a realização de laqueadura em Luzia.

Foram distribuídas as tarefas conforme a idade dos membros, sendo Renata responsável pelas tarefas domésticas e cuidado com as crianças no turno matutino e Paula no turno vespertino. Além disso, Gustavo e Júlio ficaram responsáveis pela busca do emprego formal para auxílio nas despesas da casa, a busca das verduras a cargo de Gustavo, enquanto Ester auxiliará os menores nas tarefas escolares. As consultas permaneceram sob o domínio da mãe. Durante a reunião, buscou-se responsabilizar os membros e empoderá-los quanto às necessidades familiares, como a importância da organização familiar na resolução de problemas. Verificou-se que realmente havia negligência dos integrantes da família, devido à imaturidade e pelo fato de Luzia tomar a responsabilidade para si. Abordou-se sobre a importância do acompanhamento das doenças crônicas de Luzia e Júlio, responsabilizando-os pelos tratamentos medicamentosos e consultas de rotina na ESF. Além disso, Luzia mostrou interesse pela realização de salpingectomia bilateral, portanto, foi solicitado que a mesma levasse os documentos à Unidade Básica de Saúde (UBS) para agendamento da cirurgia.

O emprego das ferramentas corroborou na compreensão da estrutura, funcionamento e dinâmica da família, aproximando os profissionais de saúde da família em estudo. Essa experiência permitiu contribuir na reorganização dos papéis familiares, encorajando a redistribuir as tarefas entre os integrantes através de um plano de cuidados elaborado a partir das demandas da família. A realização desse estudo proporcionou uma visão holística aos profissionais de saúde em relação ao cuidado, manejo e relacionamento com os pacientes, priorizando não apenas os aspectos físicos e biológicos, mas também aqueles psicossociais e econômicos, permitindo aos profissionais maior compreensão do indivíduo de acordo com o contexto no qual está inserido.

\section{REFERÊNCIAS}

1. BRASIL. Ministério da Saúde. Portaria no. 2.436 de 21 de setembro de 2017. Brasília: Diário Oficial [da] República Federativa do Brasil, 2017.

2. BRASIL. Saúde da Família: uma estratégia para a reorientação do modelo assistencial. Brasília. Ministério da Saúde, 1997. 36p.

3. BRASIL. Guia prático de matriciamento em saúde mental. Brasília: Ministério da Saúde: Centro de Estudo e Pesquisa em Saúde Coletiva, 2011.

4. BRASIL CHG. Ferramentas de acesso à Família. Residência em Medicina de Família e Comunidade e Especialização em Saúde de Família para Enfermeiros e Cirurgiões Dentistas. 2010. 18p.

5. DITTERICH RG, et al. As Ferramentas de Trabalho com Famílias Utilizadas pelas Equipes de Saúde da Família de Curitiba, PR. São Paulo: Saúde Soc., 2009; v.18, n.3, p.515-524.

6. DIAS MAS, et al. Intersetorialidade e Estratégia Saúde da Família: tudo ou quase nada a ver?. Ciência \& Saúde Coletiva, 2014; [s.I.], v. 19, n. 11, p.4371-4382.

7. FELÍCIO LFF, et al. Abordagem familiar no cuidado ao paciente com esquizofrenia: relato de caso conduzido na atenção primária à saúde. REAS, Revista Eletrônica Acervo Saúde, 2018; Vol. 10 (3), 1621-1627. 
8. FERNANDES CLC, CURRA LCD. Ferramentas de Abordagem Familiar. PROMEF.Organização SBMFC, p 13-29. Porto Alegre: Artmed/Panamericana Editora, 2006.

9. LACERDA MKS, et al. Ferramentas de abordagem familiar: estudo de uma família cadastrada em uma equipe de estratégia saúde da família em Montes Claros, Minas Gerais, Brasil. Revista de Iniciação Científica da Universidade Vale do Rio Verde, Três Corações, v. 7, n. 1, 2017, p. 25-34.

10. MELLO DF, et al. Genograma e Ecomapa: possibilidades de utilização na Estratégia de Saúde da Família. RevBrasCrescDesenvHum, 2005; v.15, n.1, p.79-89.

11. NOBRE LLR, et al. Abordagem familiar no âmbito da estratégia saúde da família: uma experiência de cuidado interdisciplinar. Revista da Universidade Vale do Rio Verde, 2014; Três Corações, v. 12, n. 2, p. 458-468.

12. OLIVEIRA PS, et al. O cuidado de um idoso frágil pela família. Rev enferm UFPE online,2016; Recife, 10(Supl. 1):273-83.

13. OLIVEIRA VCA, et al. Aplicação de ferramentas de abordagem familiar na estratégia saúde da família. RevBrasPromoç Saúde, 2017; Fortaleza, 30(4): 1-8.

14. PEREIRA APS, et al. O genograma e o ecomapa no cuidado de enfermagem em saúde da família. RevBrasEnferm, 2009; Brasília, maio-jun.; 62(3): 407-16.

15. SANTOS KKF, et al. Ferramentas de abordagem familiar: uma experiência do cuidado multiprofissional no âmbito da estratégia saúde da família. Revista da Universidade Vale do Rio Verde, 2015; Três Corações, v. 13, n. 2, p. 377-387.

16. SILVA JV, SANTOS SMR. Trabalhando com famílias utilizando ferramentas. Revista APS, 2003; 6(2):77-86. 